


\title{
Revisional Laparoscopic Mini-Gastric Bypass as a Treatment Option for Weight Loss Failure after Restrictive Procedures
}

\author{
Ahmed Adel Abbas Abdelshafy, MD; Mohamed Abdelsattar Abdelhamid, MD; Mahmoud \\ Abdelbaky Mahmoud, MD \\ Department of General Surgery, Faculty of Medicine, Ain Shams University, Egypt
}

Background: Bariatric surgery has proven its effectiveness in achieving and maintaining weight loss and improving obesity-related co-morbidities, quality of life, and survival. As demand for bariatric surgery increases, so too will the need for revisional surgeries. The revision rate following primary bariatric surgery is reported to be between $10 \%$ and $25 \%$. To facilitate weight loss surgically, many different types of bariatric procedures have been developed and established.

Objectives: To assess the effectiveness of revisional laparoscopic mini-gastric bypass for management of inadequate weight loss after different restrictive procedures.

Patients and methods: The present study is a prospective study that was conducted at Ain Shams University Hospitals in Egypt, between July 2016 and July 2018. It included Forty (40) patients who underwent a restrictive bariatric procedure 2 years ago or more with inadequate weight loss. Operative time, intraoperative complications, rate of conversion. Postoperative pain, consumption of analgesics, length of hospital stay, start of oral feeding were noted.

Results: The patients series involved 40 patients, 12 of which were male patients (40\%) whereas, 24 were female patients $(60 \%)$. The patients' age at the time of revisional surgery ranged from 23 to 57 years old with a mean \pm SD of $38.50 \pm 8.42$ years. The preoperative BMI ranged from 31.25 to $52.62 \mathrm{~kg} / \mathrm{m} 2$ with a mean \pm SD of $41.59 \pm$ $4.99 \mathrm{~kg} / \mathrm{m} 2$ with an excess weight ranging from 19.79 to $86 \mathrm{~kg}$ with a mean \pm SD of $46.51 \pm 14.27 \mathrm{~kg}$. The mean time interval between the initial restrictive surgery and the revisional LMGB was $41.63 \pm 16.92$ months. 18 patients (45\%) had a VBG as their primary restrictive surgery, 10 patients (25\%) had LAGB, 8 patients (20\%) had LSG and 4 patients (10\%) had LGCP. 28 patients (70\%) underwent LMGB for IWL compared to 12 patients (30\%) for WR.

Conclusion: The revisional Laparoscopic Mini-Gastric Bypass (r-LMGB) appears to be a feasible and safe option after failed restrictive bariatric surgery. However, additional studies with larger population and longer follow up period are required to evaluate longer-term success.

Keywords: Revisional Laparoscopic Mini-Gastric, Weight Loss Failure, Restrictive Procedures.

\section{Introduction}

Bariatric surgery has proven its effectiveness in achieving and maintaining weight loss and improving obesity-related co-morbidities, quality of life, and survival. As demand for bariatric surgery increases, so too will the need for revisional surgeries. The revision rate following primary bariatric surgery is reported to be between $10 \%$ and $25 \% .{ }^{1}$ To facilitate weight loss surgically, many different types of bariatric procedures have been developed and established. They fall into three main categories: A) Restrictive procedures that lead to fixed or adjustable physical reduction in the size of the upper gastrointestinal tract. B) Malabsorptive procedures that bypass a proportion of the intestine with less physical restriction of food intake. C) A combination of the restrictive and malabsorptive, which combines restriction of the upper food pathway with intestinal bypass. ${ }^{2}$
There are four major restrictive surgical procedures that were used over the last years after patient selection, assessment, and evaluation. These procedures are the Vertical Banded Gastroplasty (VBG) which is no longer performed nowadays, the Laparoscopic Adjustable Gastric Banding (LAGB), the Laparoscopic Greater Curvature Gastric Plication (LGCP) and the Laparoscopic Sleeve Gastrectomy (LSG). ${ }^{3}$ The Vertical Banded Gastroplasty (VBG), involved vertically partitioning the stomach at the angle of His through a window created near to the lesser curvature at the base of the pouch. A silastic ring was then placed around this window to secure the narrow, tabularized stomach reservoir. However, another technique has been adapted by some surgeons by using polypropylene mesh instead of the ring which increased the incidence of dense adhesions and subsequently adding to the technical challenge of revisional surgery. The VBG eventually was abandoned in favor of other operations such as 
the Adjustable Gastric Banding. ${ }^{4}$

The Adjustable Gastric Banding is placed around the upper stomach to create a small proximal pouch. This produces a moderate restriction in the volume and type of foods the patient is able to consume. The band also delays the emptying of the pouch, creating the sensation of satiety. ${ }^{5}$ The Laparoscopic Sleeve Gastrectomy (LSG) involves reduction of the size of the stomach by about $75 \%$ of its original size by surgically removing a large portion of it after insertion of a special bougie to calibrate a narrow tubular sleeve. ${ }^{6}$ It was found to be useful beyond its restrictive capabilities with physiologic alterations that affected metabolic processes and decreased hunger. This led to an increase of the popularity of the LSG in the late 2000s, and it rapidly replaced the LAGB as the preferred restrictive weight loss operation. $^{7}$

Laparoscopic greater curvature gastric plication (LGCP) is gaining ground in the treatment of morbid obesity, to avoid the placement of an implantable device or the irreversible resection of gastric tissue. Current technique of LGCP consists of infolding the greater curvature to reduce stomach volume by placement of rows of non-absorbable sutures. ${ }^{8}$ The reasons for revision after restrictive procedures have been reported to be unsatisfactory weight loss or intolerable adverse outcomes such as emesis, maladaptive eating syndrome, and gastroesophageal reflux disease (GERD). ${ }^{9}$ Weight loss failure after bariatric surgery is defined as achieving or maintaining less than $50 \%$ of excess weight loss (EWL) over a period of 18 to 24 months or a body mass index (BMI) of greater than 35 over the same period. ${ }^{10}$

There are specific causes of failure for each restrictive procedure, in VBG, most common causes of failure are pouch dilatation, stoma dilatation and disruption of staple line causing gastrogastric fistula. ${ }^{11}$ In LAGB, causes include band slippage, erosion into the stomach, esophageal dilation, intolerance to the device, and ultimately a decreased quality of life. ${ }^{12}$ Failure after LSG and LGCP is mainly due to dilation of the gastric lumen, this may be attributed to a lack of adequate calibration at the time of the primary procedure or a natural process of stomach tissue to dilate and become more compliant over time. ${ }^{13}$ Various revisional options have been suggested, including biliopancreatic diversion (BPD), roux en-y gastric bypass (RYGB), minigastric bypass (MGB) or reestablishing restriction. In the latter category, the options include sleeve gastrectomy and repositioning of adjustable gastric band. Although RYGB is considered by many as the gold standard revisional procedure after failed restrictive surgery, MGB is becoming an accepted alternative with satisfying outcome and similar long-term efficacy. ${ }^{14}$
The minigastric bypass (MGB) consists of two components, including, first, a long sleeved gastric tube along the lesser curvature side and, second, a Billroth type II loop gastrojejunostomy with a 200 - $250 \mathrm{~cm}$ afferent limb. Some surgeons proposed placing an anchoring suture on the afferent limb as a valve to inhibit bile reflux. ${ }^{15}$ Short and longterm results after primary laparoscopic mini-gastric bypass (MGB) have been previously reported and MGB appears to be a simple, well tolerated, and effective alternative to RYGB. However, long-term outcomes for revisional MGB for a failed restrictive procedure ( $r M G B$ ) have not yet been analyzed.

\section{Aim of the work}

Our objective in this study is to assess the effectiveness of revisional laparoscopic mini-gastric bypass for management of inadequate weight loss after different restrictive procedures.

\section{Patients and methods}

The present study is a prospective study that was conducted at Ain Shams University Hospitals in Egypt, between January 2016 and January 2018. It included Forty (40) patients who underwent a restrictive bariatric procedure 2 years ago or more with inadequate weight loss. The study was approved by the Ethics Board of Ain Shams University and an informed written consent was taken from each participant in the study.

Inclusion Criteria: Patients who underwent a restrictive bariatric surgery 2 years ago or more, with one or more of the following: Failure to achieve or maintain more than $50 \%$ of Excess Weight Loss (EWL) over a period of 2 years after restrictive bariatric surgery. Body mass index (BMI) of greater than 35 over a period of 2 years after restrictive bariatric surgery. Regain of $10 \%$ or more of the nadir weight in 1 year despite dietary counseling. Increase in Residual Gastric Volume (RGV), as assessed by radiology and/or gastroscopy.

Exclusion Criteria: Patients unfit for general anesthesia (ASA III, IV or V). Patients with failure due to intolerable adverse outcomes such as emesis, maladaptive eating syndrome, and gastroesophageal reflux disease (GERD). Patients with anatomical complications as band erosion, ring erosion, postoperative leakage. Patients with inadequate weight loss who didn't have any dietary counseling yet. Patients with psychiatric illnesses receiving anti-psychotic medications.

\section{Pre-operative assessment: \\ Full clinical history \\ Outcome assessment: Operative time, intraoperative complications, rate of conversion, postoperative pain, consumption of analgesics, length of hospital stay, start of oral feeding were}


noted. Weight loss in term of percentage excess weight loss (\%EWL), BMI at 1, 3, 6, 12, 18 and 24 month were noted. Resolution of comorbidities were noted.

\section{Statistical Analysis}

The collected data was revised, coded, tabulated and introduced to a PC using Statistical Package for Social Science (IBM Corp. Released 2011. IBM SPSS Statistics for Windows, Version 20.0. Armonk, NY: IBM Corp).

\section{Results}

Table 1: Distribution of cases according to comorbidities

\begin{tabular}{lcc}
\hline Parameter & No. & \% \\
\hline Hypertension & 8 & 20.0 \\
Type II Diabetes mellitus & 7 & 17.5 \\
Dyspnea on exertion & 32 & 80 \\
Knee pain & 23 & 57.5 \\
Back pain & 29 & 72.5 \\
Arthritis & 3 & 7.5 \\
\hline
\end{tabular}

The comorbidities (Table 1) were diabetes mellitus (type II) in 7 patients (17.5\%) all were on medical treatment, hypertension in 8 patients (20\%) all were on antihypertensive drugs, dyspnea on excretion in 32 patients $(80 \%)$, arthritis in 3 patients $(7.5 \%)$, knee pain in 23 patients $(57.5 \%)$ and back pain in 29 patients $(72.5 \%)$ all of them had continuous pain that needed medications or physiotherapy and had no serious cause.
Table 2: Early postoperative complications

\begin{tabular}{lcc}
\hline Parameter & No. & \% \\
\hline Port site infection & 0 & 0.0 \\
Bleeding & 1 & 2.5 \\
Stenosis at gastrojejunostomy & 0 & 0.0 \\
Leakage from gastric pouch staple line & 1 & 2.5 \\
Wound infection & 1 & 2.5 \\
Lung atelectasis/ chest infection & 2 & 5.0 \\
Port-site hernia & 0 & 0.0 \\
Deep venous thrombosis & 0 & 0.0 \\
Reoperation & 1 & 2.5 \\
Mortality & 0 & 0.0 \\
\hline
\end{tabular}

We had no anastomotic stenosis, port-site infection, port-site hernia or DVT in the early postoperative period. There was zero mortality in the early postoperative period (Table 2).

Table 3: Late postoperative complications

\begin{tabular}{lcc}
\hline Parameter & No. & \% \\
\hline Biliary reflux & 3 & 7.5 \\
Anemia & 5 & 12.5 \\
Hair loss & 8 & 20.0 \\
Gastrojejunostomy Stenosis/ulcer & 0 & 0.0 \\
Internal hernia & 0 & 0.0 \\
Neuropathy & 3 & 7.5 \\
Gall stones & 4 & 10.0 \\
Mortality & 0 & 0.0 \\
\hline
\end{tabular}

We had no anastomotic stenosis / ulceration or internal hernia in the late postoperative period. There was zero mortality in the late postoperative period (Table 3).

Table 4: Patients' weight in different follow up measurements

\begin{tabular}{|c|c|c|c|c|c|}
\hline & \multicolumn{2}{|c|}{ Weight } & \multirow{2}{*}{$\begin{array}{c}\text { Test } \\
\text { value* }\end{array}$} & \multirow{2}{*}{$\begin{array}{c}\text { P- } \\
\text { value }\end{array}$} & \multirow{2}{*}{ Sig } \\
\hline & Mean \pm SD & Range & & & \\
\hline Pre-operative & $117.08 \pm 16.67$ & $93-167$ & NA & NA & - \\
\hline 1 month & $112.39 \pm 16.01$ & $89.28-160.32$ & 44.412 & 0.000 & $\mathrm{HS}$ \\
\hline 3 months & $105.44 \pm 15.04$ & $83.7-150.3$ & 43.606 & 0.000 & $\mathrm{HS}$ \\
\hline 6 months & $100.88 \pm 14.44$ & $80.35-144.29$ & 38.549 & 0.000 & $\mathrm{HS}$ \\
\hline 12 months & $95.03 \pm 13.48$ & $75.53-135.63$ & 42.762 & 0.000 & $\mathrm{HS}$ \\
\hline 18 months & $91.20 \pm 12.93$ & $72.51-130.21$ & 42.708 & 0.000 & $\mathrm{HS}$ \\
\hline 24 months & $90.31 \pm 12.93$ & $71.93-129.16$ & 42.753 & 0.000 & HS \\
\hline
\end{tabular}

*: Paired t-test; HS: Highly significant; S: Significant; NS: Non significant; P-value $<0.01$ Highly significant; P-value $<0.05$ Significant; P-value > 0.05 Non significant 
Table 4 shows weight loss after the r-LMGB compared to the pre-revisonal weight throughout the 24 months follow up period; the mean weight decreased from $117.08 \mathrm{~kg} \pm 16.67 \mathrm{~kg}$ preoperatively to $112.39 \mathrm{~kg} \pm 16.01 \mathrm{~kg}$ at one month, $100.88 \mathrm{~kg} \pm$ $14.44 \mathrm{~kg}$ at six months, $95.03 \mathrm{~kg} \pm 13.48 \mathrm{~kg}$ at 12 months and reached as low as $90.31 \mathrm{~kg} \pm 12.93 \mathrm{~kg}$ at the end of the 2 years follow up period.

\section{Discussion}

As morbid obesity is a chronic, life-long disease, an effective treatment should entail a multiinterventional approach with a lifetime follow-up of intensive consultations for nutritional support, physical and psychosocial support, and reoperation if necessary. The benefits of bariatric re-interventions have to dramatically outweigh the increased adverse outcomes and the higher complication rates of revision procedures. ${ }^{16}$

Failure of adequate weight loss and weight regain has been reported to be as great as 25-30\% after restrictive bariatric procedures. Weight regain after bariatric procedures is usually multifactorial and warrants psychological and dietary assessments. The reasons for revision after restrictive procedures have been reported to be due to insufficient weight loss, weight regain, and/or surgery-related complications. ${ }^{10}$

Weight loss failure after bariatric surgery is defined as achieving or maintaining less than $50 \%$ of excess weight loss (EWL) over a period of 18 to 24 months or a body mass index (BMI) of greater than 35 over the same period. ${ }^{10}$

Different factors such as lifestyle, mental health issues, hormonal/metabolic imbalance and technical issues after surgery may contribute to failure of different restrictive bariatric procedures. Thus, patients must be investigated and treated in the specialized centers where multidisciplinary teams are available and knowledge about IWL is accumulated. The mini-gastric bypass (MGB) was introduced by Rutledge in 1997 and reported some years later. Since then, thousands of patients have been treated with this approach by several authors in different countries. ${ }^{17}$

Short and long-term results from primary laparoscopic Mini-Gastric Bypasses (LMGB) have been previously reported, and LMGB appears to be a simple, well tolerated, and effective alternative to LRYGB. However, long-term outcomes for revisional LMGB for a failed restrictive procedure ( $r$-LMGB) have not yet been heavily analyzed. ${ }^{18}$

The aim of our study was to to assess the effectiveness of revisional laparoscopic mini-gastric bypass for management of inadequate weight loss after different restrictive procedures. It is a prospective study which was done between January 2016 January 2018, at Ain Shams University Hospitals, Cairo, Egypt. The study included 40 patients who underwent a restrictive bariatric procedure 2 years ago or more with inadequate weight loss. The mean time interval between the initial restrictive surgery and the revisional LMGB was $41.63 \pm 16.92$ months. 18 patients $(45 \%)$ had a VBG as their primary restrictive surgery, 10 patients (25\%) had LAGB, 8 patients (20\%) had LSG and 4 patients $(10 \%)$ had LGCP. 28 patients (70\%) underwent LMGB for IWL compared to 12 patients (30\%) for WR.

Regarding the operative time and conversion to open surgery; in our study, the mean operative time was 118 minutes ranging between 77-188 minutes and all operations were performed laparoscopically except one case which was converted to open due to increased airway pressure with abdominal insufflation. The mean BMI of our patients was $41.59 \mathrm{~kg} / \mathrm{m} 2$.

Compared to the published results, a study conducted by Bruzzi and his colleagues (to assess 5 -year outcomes of r-LMGB after failure of restrictive surgery) included 30 patients with mean BMI of 45.5 $\mathrm{kg} / \mathrm{m} 2$, the mean operative time was 140 minutes with no conversion to open. ${ }^{19}$ Another study done by Almalki and his colleague (which compared between LMGB and LRYGB as a revision for failed restrictive procedures) included 81 patients in the r-LMGB group with a mean BMI of $37.8 \mathrm{~kg} / \mathrm{m} 2$, the mean operative time with r-LMGB was 168 with no conversion to open. ${ }^{20}$

Our outcome relatively matches the results of previous studies such as Bruzzi et al. ${ }^{19}$ who reported a BMI decrease of $15 \mathrm{~kg} / \mathrm{m} 2$ and a \%EWL of $65 \%$ after 24 months of follow-up of 30 patients who underwent r-LMGB, after failure of restrictive procedure, with a pre-revisional mean BMI of 45.5 $\mathrm{kg} / \mathrm{m} 2$.

Similarly, Almalki et al. ${ }^{20}$ study showed, 81 patients with a mean BMI of $37.8 \mathrm{~kg} / \mathrm{m} 2$ who underwent r-LMGB as a group in a comparative study between LMGB and LRYGB as a revisional procedure after failure of restrictive procedure. At 24 months postoperative, the mean BMI reached was $27.2 \mathrm{~kg} /$ $\mathrm{m} 2$ with a total BMI decrease of $10.6 \mathrm{~kg} / \mathrm{m} 2$ and a $\%$ EWL of $76.8 \%$.

The total rate of complications in our study was comparative to other studies. Our study showed a rate of $12.5 \%$ compared to $10 \%$ in Bruzzi et al. ${ }^{19}$ study and $16 \%$ in Almalki et al. ${ }^{20}$ study.

As regard the late postoperative complications; 3 cases $(7.5 \%)$ with biliary reflux were reported in 
our study and were managed successfully with conservative treatment, in contrast to Bruzzi et al. ${ }^{19}$ study that reported 2 cases $(6.66 \%)$ with intractable bile reflux that were converted to RYGB. No late complications were reported in Almalki et al. ${ }^{20}$ study.

\section{Conclusion}

The revisional Laparoscopic Mini-Gastric Bypass ( $r$-LMGB) appears to be a feasible and safe option after failed restrictive bariatric surgery. However, additional studies with larger population and longer follow up period are required to evaluate longerterm success.

\section{References}

1. Kellogg TA: Revisional bariatric surgery. Surg Clin North Am. 2011; 91(6) :1353-1371.

2. Saheba I: Manual of perioperative care, chapter 29: Bariatric Surgery, John Wiley \& Sons. 2012.

3. Moises J, Eddie G, Roderick R, et al: Failed Restrictive Surgery: Is sleeve gastrectomy a good revisional procedure? Obes Surg. 2011; 21: $157-160$.

4. Adrian G, Rebecca L: Minimally invasive bariatric surgery, chapter 4: History of bariatric and metabolic surgery, Springer, second edition. 2015.

5. O'Brien PE, McDonald L, Anderson M, Brown WA: Long term outcomes after bariatric surgery: Fifteen year follow up of adjustable gastric banding and a systematic review of the bariatric surgical literature. Ann Surg. 2013; 257(1): 87-94.

6. Stephenson JA, Al-Taan O, Wiggins T, Sutton $C D$, et al: The surgical management of obesity. InnovAiT. 2010; 3(10): 598-605.

7. Braghetto I, Csendes A, Lanzarini E, et al: Is laparoscopic sleeve gastrectomy an acceptable primary bariatric procedure in obese patients? Early and 5-year postoperative results. Surg Laparosc Endosc Percutan Tech. 2012; 22: 479486.

8. Almino R, Lyz Silva, Manoel N, Josemberg C: Laparoscopic Gastric Plication, Minimally Invasive Bariatric Surgery, Springer Science. 2015: 179-180.

9. Schouten R, Wiryasaputra DC, van Dielen FM et al: Long-term results of bariatric restrictive procedures: A prospective study. Obes Surg. 2010; 20: 1617-1626.

10. Chang VW, Lauderdale DS: Income disparity in body mass index and obesity in the United States: 1971-2002. Arch Intern Med. 2014; 165(18): 2122-2128.

11. Samir A, Eran S, Ilanit M, et al: Laparoscopic conversion of failed silastic ring vertical gastroplasty (SRVG) and vertical banded gastroplasty (VBG) into biliopancreatic diversion (BPD). J Gastrointest Surg. 2015; 19: 625-630.

12. Shen X, Zhang X, Bi J, Yin K: Long-term complications requiring reoperations after laparoscopic adjustable gastric banding: A systematic review. Surg Obes Relat Dis. 2015; 11(4) :956-64.

13. Cesana G, Uccelli M, Ciccarese $F$, et al: Laparoscopic re-sleeve gastrectomy as a treatment of weight regain after sleeve gastrectomy. World J Gastrointest Surg. 2014; 6(6): 101-6.

14. Elnahas A, Graybiel K, Farrokhyar F, Gmora S et al: Revisional surgery after failed laparoscopic adjustable gastric banding: A systematic review. Surg Endosc. 2013; 27(3): 740-5.

15. Kim Z, Hur KY (2011): Laparoscopic mini-gastric bypass for type 2 diabetes: The preliminary report. World J Surg. 2011; 35(3): 631-6.

16. Steffen R: The history and role of gastric banding. Surg Obes Relat Dis. 2008; 4(3): S713.

17. Hamdi A, Julien C, Brown $P$, et al: Midterm outcomes of revisional surgery for gastric pouch and gastrojejunal anastomotic enlargement in patients with weight regain after gastric bypass for morbid obesity. Obes Surg. 2014; 24: 13861390.

18. Lee WJ, Yu PJ, Wang W, Chen TC, et al: Laparoscopic Roux-en-Y versus mini-gastric bypass for the treatment of morbid obesity: A prospective randomized controlled clinical trial. Ann Surg. 2005; 242(1) :20-8.

19. Bruzzi M, Voron T, Zinzindohoue F, Chevallier JM, et al: Revisional single-anastomosis gastric bypass for a failed restrictive procedure: 5-year results. Surg Obes Relat Dis. 2016; 12: 240-5.

20. Almalki WJL, Jung-Chien C, Kong-Han $S$, et al: Revisional gastric bypass for failed restrictive procedures: Comparison of single-anastomosis (Mini-) and Roux-en-Y gastric bypass. Obes Surg. 2018; 28: 970-975. 